\title{
On the effect of combining cooperative communication with sleep mode
}

\author{
Thomas Demoor*, Sergey Andreev ${ }^{\dagger}$, Koen De Turck* ${ }^{*}$ Herwig Bruneel* and Dieter Fiems* \\ * Department of Telecommunications and Information Processing, Ghent University, Belgium \\ \{thdemoor,kdeturck,hb,df\}@ telin.ugent.be \\ $\dagger$ Department of Communications Engineering, Tampere University of Technology, Finland \\ sergey.andreev@tut.fi
}

\begin{abstract}
Cooperation is crucial in (next-generation) wireless networks as it can greatly attribute to ensuring connectivity, reliability, performance, ... Relaying looks promising in a wide variety of network types (cellular, ad-hoc on-demand), each using a certain protocol. Energy efficiency constitutes another key aspect of such networks, as battery power is often limited, and is typically achieved by sleep mode operation. As the range of applications is very broad, rather than modelling one of the protocols in detail, we construct a high-level model capturing the two essential characteristics of cooperation and energy efficiency: relaying and sleep mode, and study their interaction. The used analytical approach allows for accurate performance evaluation and enables us to unveil less trivial trade-offs and to formulate rules-of-thumb applicable across all potential scenarios.
\end{abstract}

\section{INTRODUCTION AND BACKGROUND}

It is evident that cooperative communications allow for a flexible and robust exchange of information and hence they receive increasing attention from the research community. Historically, the core ideas behind cooperative communications were introduced in the fundamental work [1], where the capacity of a three-node network was studied, with one of the nodes acting as a relay. A overview of the rapidly expanding literature on cooperative communications may be found in [2].

In [3] the scenario is considered where the direct link from the originator is not available due to e.g. its poor quality. As such, the originator can only communicate its packets to the destination through the relays and [3] proposes an efficient protocol for the relay selection. Motivated by the worst-case scenario from [3], we consider the case where the centralized controller can only receive packets from the relay user, thus assuming no direct link. This scenario is also practically relevant to contemporary 3GPP relay systems for back-haul offloading in frequency or space [4]. Also, [5] suggests that wireless clients themselves may relay data for other clients thus acting as (mobile) relay stations. Developing this concept of client relays, [6], [7] propose a simple cooperation protocol and analyse the basic trade-offs behind client relay functionality. Furthermore, this is also relevant for novel advanced wireless services, including machine-tomachine (M2M) communications [8].

Another practical aspect of cellular networking is that mobile users are typically equipped with limited battery power. During time intervals when no packets are being sent or received, a wireless client may start a sleep period by shutting down all transmit and receive activities and thus save power. Novel communication standards [4] and [9] support reductions in client power consumption through various sleep mode mechanisms. Over recent years, an extensive literature on sleep mode operation has accumulated. The vast majority of research papers consider power saving schemes for IEEE 802.16 technology. The legacy IEEE 802.16e sleep mode operation for both downlink and uplink traffic was considered in [10], [11] and IEEE $802.16 \mathrm{~m}$ was studied in [12]. A more practical comparison of these two may be found in [13]. In 3GPP LTE and LTE-Advanced, the equivalent power saving scheme is termed discontinuous reception (DRX). The operation of DRX has been a subject to fewer research. Some analytical results for DRX in case of bi-directional traffic were also presented in [14]. Our model can be tailored to either IEEE 802.16 sleep mode, or 3GPP DRX mode operation.

The current contribution couples power saving and cooperative user behaviour. Consider a relay, an originator and a destination. Both the relay and the originator receive packets for the destination but only the relay can send. The originator is not connected to the destination but its packets can be transferred to the relay. However, the relay only cooperates if its own queue is empty. Furthermore, the relay adopts a sleep mode mechanism. If the relay queue is empty and the originator has packets to be relayed, all packets in the originator queue are transferred to the relay queue. If both the relay and the originator queues are empty, the relay turns off its communication circuitry and enters sleep mode for some predefined (possibly random) time. At the end of this period, if the relay queue is empty, a subsequent (possibly different) sleep period is started. Otherwise, communication possibilities are re-established and normal operation is resumed.

The developed model is intentionally broad-scoped hence capturing only the key aspects of such systems making it applicable to different technical implementations. Through its performance analysis, we aim at furthering understanding fundamental aspects of systems with cooperation and sleep mode mechanisms. The rest of the text is organized as follows. The next section defines the mathematical details of the model. The performance of this model is assessed in section III. Next, the results are discussed from a practical point of view and the final section draws conclusions. 


\section{QUEUEING MODEL}

Consider a queueing system in discrete-time, i.e. time is divided into fixed length slots and arrivals and departures are synchronised to slot boundaries. The queueing system at hand consists of two queues, termed relayer and originator queue, each having unlimited capacity for storing packets. Let $A_{1, k}$ and $A_{2, k}$ denote the number of arriving packets at the $k$ th slot boundary in the relayer and originator queue, respectively. The consecutive $\left(A_{1, k}, A_{2, k}\right)$ constitute a sequence of independent and identically distributed $\mathbb{N}^{2}$-valued random variables. Let $A\left(z_{1}, z_{2}\right)$ denote the common joint probability generating function of this sequence, $A\left(z_{1}, z_{2}\right)=\mathrm{E}\left[z_{1}^{A_{1}} z_{2}^{A_{2}}\right],\left(A_{1}, A_{2}\right)$ being a generic version of $\left(A_{1, k}, A_{2, k}\right)$.

While there are two queues, there is only one server which only serves packets in the relayer queue in order of arrival. Let $S_{k}$ denote the service time (in slots) of the $k$ th packet in the relayer queue. The consecutive $S_{k}$ constitute a sequence of independent and identically distributed $\mathbb{N}^{*}$-valued random variables; let $S(z)$ denote their common probability generating function and let $S$ denote a generic service time. When the relayer queue is empty, it checks for packets in the originator queue. If there are such packets, they immediately move to the relayer queue and the server starts transmitting these packets. If not, the server enters so-called sleep-mode. During sleepmode, the server does not constantly check the relayer queue for new packet arrivals. Instead, the server wakes up after some (possibly random) time, and checks only the relayer. If there are packets in the relayer queue, the server returns from sleepmode and starts serving packets again. If there are none, the server goes to sleep again, wakes up after some time, etc. The lengths (in slots) of the consecutive sleep periods constitutes a sequence of independent random variables, the distribution depending on the number of sleep-periods already taken. Let $T_{i}$ denote the length of the $i$ th consecutive sleep-period with corresponding probability generating function $T_{i}(z)$. Moreover, we assume there exists some $K$ such that $T_{i}(z)=T_{K}(z)$ for $i>K$, as is the case in practical applications.

\section{QUEUEING ANALYSIS}

\section{A. Queue content at departure epochs}

Let $U_{1, k}$ and $U_{2, k}$ denote the number of packets in the relayer and originator queue upon departure of the $k$ th packet. Furthermore, let $A_{1, k}^{j}$ and $A_{2, k}^{j}$ represent the number of relayer and originator packet arrivals during the $j$ th service slot of the $k$ th packet and let $V_{1, k}$ and $V_{2, k}$ indicate the number of relayer and originator packets arriving during the entire sleep period.

We first relate the number of packets in the system at consecutive departure epochs. If the relayer queue is not empty upon departure of packet $k\left(U_{1, k}>0\right)$, transmission of the next packet starts immediately. Hence, at the $k+1$ st departure epoch, each queue contains all packets that arrived during the transmission time of the $k+1$ st packet, whereas the $k+1$ st packet itself has departed from the relayer queue. This implies,

$$
U_{1, k+1}=U_{1, k}-1+\sum_{j=1}^{S_{k+1}} A_{1, k}^{j}, U_{2, k+1}=U_{2, k}+\sum_{j=1}^{S_{k+1}} A_{2, k}^{j}
$$

When the relayer queue is empty upon departure of packet $k\left(U_{1, k}=0\right)$ but the originator queue is not $\left(U_{2, k}>0\right)$, all packets of the originator queue immediately move to the relayer and transmission of the next packet starts. This packet leaves at the next departure epoch and arrivals during its transmission time are stored in the queues. That is,

$$
U_{1, k+1}=U_{2, k}-1+\sum_{j=1}^{S_{k+1}} A_{1, k}^{j}, U_{2, k+1}=\sum_{j=1}^{S_{k+1}} A_{2, k}^{j}
$$

Finally, if both relayer and originator queue are empty $\left(U_{1, k}=\right.$ $U_{2, k}=0$ ), the relaying node goes into sleep mode and only wakes up when there are packets in the relayer queue. Then, the next packet is transmitted.

$$
U_{1, k+1}=V_{1, k}-1+\sum_{j=1}^{S_{k+1}} A_{1, k}^{j}, U_{2, k+1}=V_{2, k}+\sum_{j=1}^{S_{k+1}} A_{2, k}^{j}
$$

Next, we determine $V\left(z_{1}, z_{2}\right)$, the joint pgf of $V_{1, k}$ and $V_{2, k}$, tracking arrivals during an entire sleep period. For notational purposes, let $T_{i}^{*}\left(A\left(z_{1}, z_{2}\right)\right)=T_{i}\left(A\left(z_{1}, z_{2}\right)\right)-T_{i}\left(A\left(0, z_{2}\right)\right)$. Conditioning on the number of sleep periods produces

$$
\begin{aligned}
V\left(z_{1}, z_{2}\right)= & \sum_{i=1}^{K-1} T_{i}^{*}\left(A\left(z_{1}, z_{2}\right)\right) \prod_{j=1}^{i-1} T_{j}\left(A\left(0, z_{2}\right)\right) \\
& +\frac{T_{K}^{*}\left(A\left(z_{1}, z_{2}\right)\right)}{1-T_{K}\left(A\left(0, z_{2}\right)\right)} \prod_{j=1}^{K-1} T_{j}\left(A\left(0, z_{2}\right)\right) .
\end{aligned}
$$

as, if the $i$ th sleep period is the final one, packets have arrived in the relayer queue during that period but not during the $i-1$ previous ones and $T_{i}(z)=T_{K}(z)$ for $i>K$.

If, on average, the arrivals do not exceed the service capacity, i.e. if $\rho=\mathrm{E}[S]\left(\mathrm{E}\left[A_{1}\right]+\mathrm{E}\left[A_{2}\right]\right)<1$, a stationary distribution exists. Let $U_{d}\left(z_{1}, z_{2}\right)$ denote the joint probability generating function of the stationary relayer and originator queue content upon departure of a packet. The system equations (1) to (3) then correspond to

$$
\begin{aligned}
& U_{d}\left(z_{1}, z_{2}\right)=S\left(A\left(z_{1}, z_{2}\right)\right) \\
& \times \frac{U_{d}\left(0, z_{1}\right)-U_{d}\left(0, z_{2}\right)-U_{d}(0,0)\left(1-V\left(z_{1}, z_{2}\right)\right)}{z_{1}-S\left(A\left(z_{1}, z_{2}\right)\right)} .
\end{aligned}
$$

with remaining unknowns $U_{d}(0, z)$ and $U_{d}(0,0)$. Plugging in $\left(z_{1}, z_{2}\right)=(1,1)$ and invoking normalisation yields,

$$
U_{d}(0,0)=\frac{1-\mathrm{E}[S]\left(\mathrm{E}\left[A_{1}\right]+\mathrm{E}\left[A_{2}\right]\right)}{\mathrm{E}\left[V_{1}\right]+\mathrm{E}\left[V_{2}\right]},
$$

where $\mathrm{E}\left[V_{1}\right]=\left.\frac{d}{d z} V(z, 1)\right|_{z=1}$ and $\mathrm{E}\left[V_{2}\right]=\left.\frac{d}{d z} V(1, z)\right|_{z=1}$ denote the mean number of packets in relayer and originator buffer respectively, upon returning from sleep mode. 
By Rouché's theorem, one can show that for each $z$ inside the unit disk, there exists a value $\chi(z)$ such that

$$
\chi(z)=S(A(\chi(z), z)) .
$$

Since $U_{d}\left(z_{1}, z_{2}\right)$ is analytic inside the unit disk and the numerator of the right-hand side of (5) equals zero for $\left(z_{1}, z_{2}\right)=(\chi(z), z)$ for $z$ inside the unit disk, the denominator must also equal zero yielding

$$
U_{d}(0, z)=U_{d}(0, \chi(z))-U_{d}(0,0)(1-V(\chi(z), z))
$$

Repeated application of the functional equation yields

$$
U_{d}(0, z)=U_{d}(0,1)-U_{d}(0,0) \sum_{i=0}^{\infty}\left(1-V\left(\chi_{i+1}(z), \chi_{i}(z)\right)\right) .
$$

with $\chi_{0}(z)=z$ and $\chi_{i}(z)=\chi\left(\chi_{i-1}(z)\right)$. Plugging in $z=0$ further yields,

$$
U_{d}(0,1)=U_{d}(0,0)\left[1+\sum_{i=0}^{\infty}\left(1-V\left(\chi_{i+1}(0), \chi_{i}(0)\right)\right)\right] .
$$

Combining these expressions, finally delivers,

$$
\begin{aligned}
& U_{d}(0, z)=U_{d}(0,0) \\
& \times\left[1+\sum_{i=0}^{\infty}\left(V\left(\chi_{i+1}(z), \chi_{i}(z)\right)-V\left(\chi_{i+1}(0), \chi_{i}(0)\right)\right)\right]
\end{aligned}
$$

such that,

$$
\begin{gathered}
U_{d}\left(z_{1}, z_{2}\right)=\frac{1-\rho}{\mathrm{E}\left[V_{1}\right]+\mathrm{E}\left[V_{2}\right]} \frac{S\left(A\left(z_{1}, z_{2}\right)\right)}{\left(z_{1}-S\left(A\left(z_{1}, z_{2}\right)\right)\right.} \\
\times\left[\sum_{i=0}^{\infty}\left[V\left(\chi_{i+1}\left(z_{1}\right), \chi_{i}\left(z_{1}\right)\right)-V\left(\chi_{i+1}\left(z_{2}\right), \chi_{i}\left(z_{2}\right)\right)\right]\right. \\
\left.-\left(1-V\left(z_{1}, z_{2}\right)\right)\right] .
\end{gathered}
$$

\section{B. System state at random slot boundaries}

We can now obtain the joint probability generating function of the system state (in the Markovian sense) at random slot boundaries. During sleep periods, the queueing system is described by (i) the remaining length of the on-going sleep period, (ii) the ordinal number of the sleep period, and (iii) the number of packets in each queue. During busy periods, the system state is captured by (i) the remaining transmission time and (ii) the number of packets in each queue.

Consider the queue content during a (randomly tagged) busy slot. Let $H$ denote the remaining number of transmission slots after the tagged slot and let $U_{1}$ and $U_{2}$ denote the number of packets in the relayer and originator queue respectively. For ease of notation, let $Q$ be a random variable which equals 0 if the system is busy during the tagged slot and $i$ if it is during the $i$ th consecutive sleep period. The (partial) joint probability generating function of the system state during busy periods is then given by $P_{b}\left(y, z_{1}, z_{2}\right)=\mathrm{E}\left[y^{H} z_{1}^{U_{1}} z_{2}^{U_{2}} 1\{Q=0\}\right]$. By standard $z$-transform techniques, we find,

$$
P_{b}\left(y, z_{1}, z_{2}\right)=\rho \frac{U_{d}\left(z_{1}, z_{2}\right) z_{1}}{S\left(A\left(z_{1}, z_{2}\right)\right)} \frac{\mathrm{E}\left[\sum_{i=0}^{S-1} A\left(z_{1}, z_{2}\right)^{i} y^{S-i}\right]}{S^{\prime}(1)}
$$

Obviously, a random slot is a busy slot with probability $\rho$. The second factor in the expression above is the probability generating function of the relayer and originator queue content at the beginning of a slot where a packet transmission starts. The third factor then accounts for state changes during the transmission. The former expression simplifies to,

$$
\begin{aligned}
P_{b}\left(y, z_{1}, z_{2}\right) & =\left(\mathrm{E}\left[A_{1}\right]+\mathrm{E}\left[A_{2}\right]\right) \\
& \times \frac{U_{d}\left(z_{1}, z_{2}\right) z_{1} y}{S\left(A\left(z_{1}, z_{2}\right)\right)} \frac{S\left(A\left(z_{1}, z_{2}\right)\right)-S(y)}{A\left(z_{1}, z_{2}\right)-y} .
\end{aligned}
$$

Now, consider the queue content during a randomly tagged sleep slot, and let $G$ denote the remaining number of slots till the sleep period ends. The partial joint probability generating function of the system state during sleep-periods then is

$$
\begin{aligned}
& P_{v}\left(y, z_{1}, z_{2} ; i\right)=\mathrm{E}\left[y^{G} z_{1}^{U_{1}} z_{2}^{U_{2}} 1\{Q=i\}\right] \\
& \quad=\kappa_{i} \frac{\prod_{j=1}^{i-1} T_{j}\left(A\left(0, z_{2}\right)\right)}{\prod_{j=1}^{i-1} T_{j}(A(0,1))} \frac{T_{i}\left(A\left(z_{1}, z_{2}\right)\right)-T_{i}(y)}{T_{i}^{\prime}(1)\left(A\left(z_{1}, z_{2}\right)-y\right)} y .
\end{aligned}
$$

with,

$$
\kappa_{i}=(1-\rho) \frac{T_{i}^{\prime}(1) \prod_{j=1}^{i-1} T_{j}(A(0,1))}{\sum_{k=1}^{\infty} T_{k}^{\prime}(1) \prod_{j=1}^{k-1} T_{j}(A(0,1))} .
$$

Here $\kappa_{i}$ denotes the probability that a random tagged slot is a slot during a sleep period with ordinal number $i$. Further, the second factor equals the probability generating function of the (originator) queue content at the beginning of the $i$ th sleep period. Finally, the third factor accounts for the state changes during the sleep period.

\section{RESUlts}

We apply the results obtained in the previous sections to a practical scenario analogous to the recommendations of [15]. We assume a frame (slot) length of $5 \mathrm{~ms}$, and a fixed size of WiMAX packets of 200 bytes. This results in a maximal throughput of $320 \mathrm{kbps}$. 'Packets' arriving at the originator and the relayer queue represent web documents. The service time of such a packet is $m=\lceil M / 200\rceil$ with $M$ the document size in bytes which has a lognormal distribution with a mean and standard deviation of $10.71 \mathrm{kB}$ and $25.03 \mathrm{kB}$ respectively, subsequently be truncated to $100<M<2 \cdot 10^{6}$ and renormalised. Then, $S(z)$ is a polynomial of degree $2 \cdot 10^{6} / 200$. We assume that the documents arrive at the relayer and originator queue according to a process with pgf $A\left(z_{1}, z_{2}\right)=\left(1+\lambda_{1} / 2 *\left(z_{1}-1\right)+\lambda_{2} / 2 *\left(z_{2}-1\right)\right)^{2}$.

In figs. 1 and 2 , we show the mean buffer content of both queues, with constant repeated sleep periods of respectively 100 and 1000 slots and where the load of one type kept constant at $20 \%$, while varying the load of the other type. In fig. 1, 


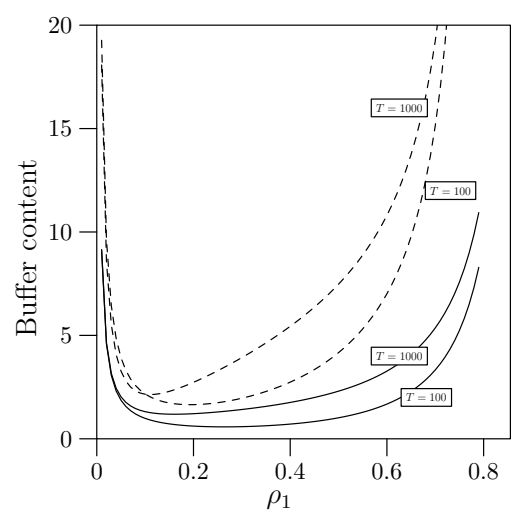

Fig. 1. Mean buffer content versus the load $\rho_{1}$ of the relayer queue, with sleep mode parameter $T=100$ and $T=1000$. Dashed lines represent the relayer buffer content, while full lines represent the originator buffer content.

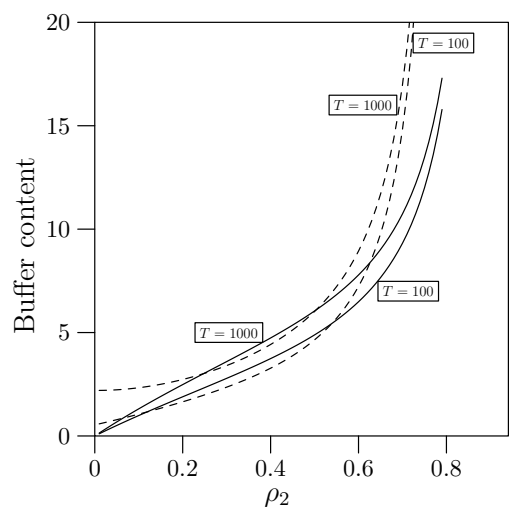

Fig. 2. Mean buffer content versus the load $\rho_{2}$ of the originator queue, with sleep mode parameter $T=100$ and $T=1000$. Dashed lines represent the relayer buffer content, while full lines represent the originator buffer content.

the average queue content of all types decreases at first because more relayer packets means less sleep mode. As the relayer load increases further, the queueing effect appears and packets accumulate in the queue. Surprisingly, for low loads longer sleep modes lead to lower queue content. As the the load increases, longer sleep periods evidently instigate higher buffer contents. Futhermore, the U-shape of the curves indicates that an optimal situation is apparent, although different for relay and originator. Consequently, this opens the door to abuse as, when the load is low, "dummy" packets could be sent to reach the optimum, of course at the cost of the power savings performance of the relay. This raises the need for policing in environments with relays. Furthermore, the (negative) effect of longer sleep periods on queue content is apparent, although the effect on power savings is evidently positive. Next, consider fig. 2. Evidently, the queue content of each type increases with the originator load. This respectively because of the queueing effect and because all packets eventually pass through the relay. Also, the effect of the length of the sleep period is clearly apparent. However, notice that for moderate load, on average, most packets reside in the originator. Again, the length of the sleep periods plays a crucial role.

\section{CONClusion}

In this paper, we have analysed the joint impact of two important mechanisms of modern wireless networks: a relay mechanism enabling a mobile device to transmit data via a relayer node, combined with a sleep mode mechanism that allows the relayer queue to save energy by temporarily switching off the radio interface. By adjusting the input processes, a great range of different technical implementations can be studied in detail. This wide-applicability is possible because the model only captures the key aspects of the system. We obtain closedfrom expressions for the moments of buffer content. Numerical results illustrate the trade-off between performance and power savings. Also, we uncover some less trivial aspects of system operation and identify the need for policing of traffic for the protocols to be always feasible in practice.

\section{ACKNOWLEDGMENT}

The final author acknowledges the support of Research Foundation - Flanders (FWO-Vlaanderen).

\section{REFERENCES}

[1] T. Cover and A. E. Gamal, "Capacity theorems for the relay channel," IEEE Transactions on Information Theory, vol. 25, no. 5, pp. 572-584, 1979.

[2] A. Nosratinia, T. Hunter, and A. Hedayat, "Cooperative communication in wireless networks," IEEE Communications Magazine, vol. 42, no. 10, pp. 74-80, 2004.

[3] R. Tannious and A. Nosratinia, "Spectrally-efficient relay selection with limited feedback," IEEE Journal on Selected Areas in Communications, vol. 26, no. 8, pp. 1419-1428, 2008.

[4] LTE Release 10 \& beyond (LTE-Advanced).

[5] J. Sydir and R. Taori, "An evolved cellular system architecture incorporating relay stations," IEEE Communications Magazine, vol. 47, no. 6, pp. 115-121, 2009.

[6] S. Andreev, O. Galinina, and A. Vinel, "Performance evaluation of a three node client relay system," International Journal of Wireless Networks and Broadband Technologies, vol. 1, pp. 73-84, 2011.

[7] S. Andreev, O. Galinina, and Y. Koucheryavy, "Energy-efficient client relay scheme for machine-to-machine communication," in Proc. of the IEEE Global Telecommunications Conference, 2011.

[8] G. Wu, S. Talwar, K. Johnsson, N. Himayat, and K. Johnson, "M2M: From mobile to embedded Internet," IEEE Communications Magazine, vol. 49, no. 4, pp. 36-43, 2011.

[9] IEEE 802.16m-2011, Amendment to IEEE Standard for Local and metropolitan area networks. Advanced Air Interface.

[10] K. D. Turck, S. Andreev, S. D. Vuyst, D. Fiems, S. Wittevrongel, and H. Bruneel, "Performance of the IEEE 802.16e sleep mode mechanism in the presence of bidirectional traffic," in Proc. of the GreenComm Workshop. IEEE International Conference on Communications, 2009.

[11] S. D. Vuyst, K. D. Turck, D. Fiems, S. Wittevrongel, and H. Bruneel, "Delay versus energy consumption of the IEEE 802.16e sleep-mode mechanism," IEEE Transactions on Wireless Communications, vol. 8, no. 11 , pp. 5383-5387, 2009.

[12] S. Baek and B. Choi, "Performance analysis of sleep mode operation in IEEE $802.16 \mathrm{~m}$ with both uplink and downlink packet arrivals," in Proc. of the CAMAD Workshop. IEEE International Conference on Communications, 2011, pp. 112-116.

[13] A. Anisimov, S. Andreev, O. Galinina, and A. Turlikov, "Comparative analysis of sleep mode control algorithms for contemporary metropolitan area wireless networks," in Proc. of the International Conference on Next Generation Wired/Wireless Advanced Networking, 2010, pp. 184-195.

[14] S. Baek and B. Choi, "Analysis of discontinuous reception (DRX) with both downlink and uplink transmissions in 3GPP LTE," in Proc. of the International Conference on Queueing Theory and Network Applications, 2011.

[15] IEEE 802.16m-08/004r5, IEEE 802.16m Evaluation Methodology Document, Jan. 2009. 